\title{
Altered Blood Flow in the Ophthalmic and Internal Carotid Arteries in Patients with Age-Related Macular Degeneration Measured Using Noncontrast MR Angiography at 7T
}

(D) M.L. Hibert, (D) Y.I. Chen, (D) N. Ohringer, (DW.J. Feuer, (D).K. Waheed, (D).S. Heier, (D) M.W. Calhoun, DP.J. Rosenfeld, and

(D).R. Polimeni

O- $\equiv$

\begin{abstract}
BACKGROUND AND PURPOSE: Age-related macular degeneration is associated with reduced perfusion of the eye; however, the role of altered blood flow in the upstream ophthalmic or internal carotid arteries is unclear. We used ultra-high-field MR imaging to investigate whether the diameter of and blood flow in the ophthalmic artery and/or the ICA are altered in age-related macular degeneration and whether any blood flow changes are associated with disease progression.
\end{abstract}

MATERIALS AND METHODS: Twenty-four patients with age-related macular degeneration and 13 similarly-aged healthy controls participated. TOF and high-resolution dynamic 2D phase-contrast MRA $\left(0.26 \times 0.26 \times 2 \mathrm{~mm}^{3}, 100\right.$-ms effective sampling rate) was acquired at 7T. Vessel diameters were calculated from cross-sectional areas in phase-contrast acquisitions. Blood flow time-series were measured across the cardiac cycle.

RESULTS: The ophthalmic artery vessel diameter was found to be significantly smaller in patients with age-related macular degeneration than in controls. Volumetric flow through the ophthalmic artery was significantly lower in patients with late age-related macular degeneration, with a significant trend of decreasing volumetric ophthalmic artery flow rates with increasing disease severity. The resistance index was significantly greater in patients with age-related macular degeneration than in controls in the ophthalmic artery. Flow velocity through the ophthalmic artery and ICA was significantly higher in patients with age-related macular degeneration. Ophthalmic artery blood flow as a percentage of ipsilateral ICA blood flow was nearly double in controls than in patients with age-related macular degeneration.

CONCLUSIONS: These findings support the hypothesis that vascular changes upstream to the eye are associated with the severity of age-related macular degeneration. Additional investigation into the potential causality of this relationship and whether treatments that improve ocular circulation slow disease progression is warranted.

ABBREVIATIONS: AMD = age-related macular degeneration; $\mathrm{EC}=$ eddy current; GEE = generalized estimating equation; OA $=$ ophthalmic artery; $\mathrm{PC}=$ phase-contrast; VENC = encoding velocity

$\mathbf{A}_{\mathrm{p}}^{\mathrm{s}}$ ge-related macular degeneration (AMD) is a late-onset complex genetic disease-and the leading cause of blindness

Received December 21, 2020; accepted after revision April 4, 2021.

From the Athinoula A. Martinos Center for Biomedical Imaging (M.L.H., Y.I.C., N.O., J.R.P.), Massachusetts General Hospital, Charlestown, Massachusetts; Department of Radiology (Y.I.C., J.R.P.), Harvard Medical School, Boston, Massachusetts; Department of Ophthalmology (W.J.F., P.J.R.), Bascom Palmer Eye Institute, University of Miami Miller School of Medicine, Miami, Florida; New England Eye Center (N.K.W.), Tufts Medical Center, Boston, Massachusetts; Ophthalmic Consultants of Boston (J.S.H.), Boston, Massachusetts; OcuDyne Inc (M.W.C.), Roseville, Minnesota; and Harvard-Massachusetts Institute of Technology Division of Health Sciences and Technology (J.R.P.), Massachusetts Institute of Technology, Cambridge, Massachusetts.

This work was supported, in part, by the National Institutes of Health (National Institute of Biomedical Imaging and Bioengineering grants P41-EB015896 and R01EB019437), the BRAIN Initiative (National Institute of Mental Health grant R01-MH111419), and the Massachusetts General Hospital/Harvard-Massachusetts Institute of Technology Division of Health Sciences and Technology Athinoula A. Martinos Center for Biomedical Imaging and was made possible by the resources provided by National Institutes of Health Shared Instrumentation Grants S10-RR023401, S10-RR019307, S10RR023043, S10-RR019371, and S10-RR020948. It was also supported by OcuDyne Inc. among the elderly worldwide-caused by irreversible damage to the macula, resulting in central vision loss. ${ }^{1,2}$ AMD is divided into 3 stages known as early, intermediate, and late. ${ }^{3}$ Early and intermediate AMD are characterized by drusen-lipoprotein deposits under the retinal pigment epithelium-which increase in size along with pigment deposition, while late AMD is characterized by either the presence of exudation caused by macular neovascularization or the formation of macular atrophy known as geographic atrophy. Due to their clinical features, nonexudative

\footnotetext{
Please address correspondence to Jonathan R. Polimeni, PhD, Athinoula A. Martinos Center for Biomedical Imaging, Massachusetts General Hospital, 149 Thirteenth St, Suite 2301, Charlestown, MA, 02129; e-mail: jonp@nmr.mgh.harvard.edu

- Indicates open access to non-subscribers at www.ajnr.org

Indicates article with online supplemental data.

http://dx.doi.org/10.3174/ajnr.A7187
} 
AMD is also referred to as dry AMD, while exudative or neovascular AMD is referred to as wet AMD. Approximately 15\% of AMD progresses to the exudative form of the disease, which can be treated with intravitreal injections of vascular endothelial growth factor inhibitors, but there are no effective treatment options for dry AMD regardless of stage. ${ }^{4}$

The choroid is the vascular layer that provides most of the nutritional support to the outer retina and retinal pigment epithelium, which are the layers primarily affected in the macula in AMD. Prior studies have demonstrated reduced choroidal perfusion in patients with AMD, measured with Doppler imaging ${ }^{5,6}$ and more recently with MR imaging-based perfusion measures. ${ }^{7}$ Reduced choroidal perfusion was found to correlate to AMD severity $^{6}$ and also the risk of developing wet AMD. ${ }^{8}$ In addition, risk factors for developing AMD include cardiovascular disease and a history of smoking. ${ }^{9}$ These studies indicate an important link between blood flow and development and progression of AMD. It is unclear whether the vasculature upstream to the choroid is altered in patients with AMD.

In this report, we investigated whether vessel diameters and blood flow in the ophthalmic artery (OA) or the internal carotid artery (ICA), the first two arteries upstream of the choroid, are altered in AMD. We used 7T MRA to obtain high-resolution measures of $\mathrm{OA}$ and ICA blood flow and morphometry without the use of intravascular contrast agents. Here 7T MRA was chosen over lower magnetic field strengths due to its higher SNR, which enables imaging at the higher spatial resolution with sufficient SNR to reliably assess vessels with small cross-sectional diameters such as the OA. ${ }^{10,11}$ This imaging protocol has the potential to detect abnormalities in the relevant vascular anatomy and associated blood flow in individual patients. This work will lead to a better understanding of the vascular changes beyond the choroid and may aid in the development of new treatment strategies for both wet and dry AMD.

\section{MATERIALS AND METHODS \\ Study Participants}

Subjects with different stages of AMD and similarly aged controls participated in this study, approved by the Partners Human Research Committee, after providing written informed consent. Patients were referred from two Boston-area ophthalmologists who provided patients' diagnostic information, and the stages of AMD were confirmed by two retina specialists (N.K.W. and P.J.R.). Healthy controls were identified using advertisements in the greater Boston area and also included spouses of patients enrolled in the study. Exclusion criteria for both patients and controls included contraindications for MR imaging, color vision deficiency/color blindness, and a history of neurologic or psychiatric illnesses. Some patients had a different stage of AMD in each eye. In all cases, left and right OAs and ICAs were scanned and analyzed separately. Images with head motion artifacts were excluded.

\section{MR Imaging Data Acquisition}

All subjects were scanned on a whole-body 7T Magnetom MR imaging scanner (Siemens) using a 32-channel head receive array coil with a birdcage transmit coil built in-house. ${ }^{12}$ We performed local $\mathrm{B}_{0}$ shimming in the region around the OAs using a highresolution local $\mathrm{B}_{0}$ field map (WIP 452B; Siemens). A 0.5-mm isotropic 3D-TOF-MRA scan covering the OAs and carotid siphon was acquired for each subject with the following parameters: $\mathrm{TR} / \mathrm{TE}=21 / 1.83 \mathrm{~ms}$, flip angle $=45^{\circ}$, slab thickness $=$ $24 \mathrm{~mm}$, bandwidth $=766 \mathrm{~Hz} /$ pixel, acceleration factor $=2$, acquisition time $=8$ minutes 44 seconds. To minimize the TE value, we disabled flow compensation; with a sufficiently low TE value, streaking or displacement artifacts due to pulsatile or steady flow, respectively, are minimized, reducing the need for flow compensation. During protocol development, it was found that the ultrashort TE of $1.83 \mathrm{~ms}$ and an asymmetric echo factor of $36 \%$ were critical to obtaining sufficient SNR to visualize the entire path of the OA.

Blood flow estimation for each OA was performed with a single-slice high-resolution 2D dynamic quantitative phasecontrast (PC) MRA acquisition. The 3D-TOF images were used to carefully position the PC acquisitions orthogonal to the trajectory of each $\mathrm{OA}$ and approximately $1.5 \mathrm{~cm}$ distal to the ostium and before any branches of the OA (Fig 1), along the distal segment of the OA past "angle b," ${ }^{13}$ the second bend in the OA. The PC-MRA acquisitions used a single velocity encoding direction and multiple encoding velocities (VENCs) with the following parameters: $0.26 \times 0.26 \times 2 \mathrm{~mm}^{3}$ voxels and TR/TE/flip angle $=$ $34.2 / 5.39 \mathrm{~ms} / 15^{\circ}$. Acquisition times varied on the basis of subjects' heart rates but typically lasted approximately 8-10 minutes. To enable straightforward identification of the OA without image registration, we centered the FOV of each PC-MRA acquisition on the OA.

Blood flow estimation in each ICA followed a similar protocol, positioning each 2D-PC-MRA acquisition below the carotid siphon. Due to the larger vessel size, we opted for a lower inplane resolution $\left(0.5 \times 0.5 \times 2 \mathrm{~mm}^{3}\right)$ with $\mathrm{TR} / \mathrm{TE} /$ flip angle $=$ $27.2 / 3.9 \mathrm{~ms} / 15^{\circ}$. Acquisition times varied on the basis of the subjects' heart rates but typically lasted approximately 4-5 minutes.

PC-MRA acquisitions were cardiac-gated prospectively, using the MR imaging system vendor's built-in pulse oximeter trigger, and had 3 VENCs, which were typically 25,45 , and $70 \mathrm{~cm} / \mathrm{s}$ for $\mathrm{OA}$ acquisitions and 45,70 , and $110 \mathrm{~cm} / \mathrm{s}$ for ICA acquisitions. Pilot scans indicated that these values would be appropriate for these two vessels of interest in most subjects and that the optimal VENC for dynamic analysis varied among subjects. Additional PC-MRA acquisitions with adjusted VENCs were included when an individual subject's peak flow rates were observed to be outside the default VENC range. Due to cardiac rate variations across participants, there was minor variability in the temporal resolution of the blood flow acquisition; on average, 29.7 (SD, 5.2) samples were acquired during the cardiac cycle, and the average cycle duration was 981 (SD, 164) ms, yielding an average sampling rate of $33(\mathrm{SD}, 6) \mathrm{ms}$. All analyses were performed on image data reconstructed online using the vendor-supplied software.

\section{Eddy Current Correction}

Due to the relatively large gradient strengths used for velocity encoding, VENC-specific spatially varying phase changes could be induced by eddy currents (ECs) during the acquisition. These unwanted phase effects could cause artifactual differences in the estimated phase, increasing or decreasing the calculated flow velocities from the true velocity. ${ }^{14}$ Thus, these EC biases were 

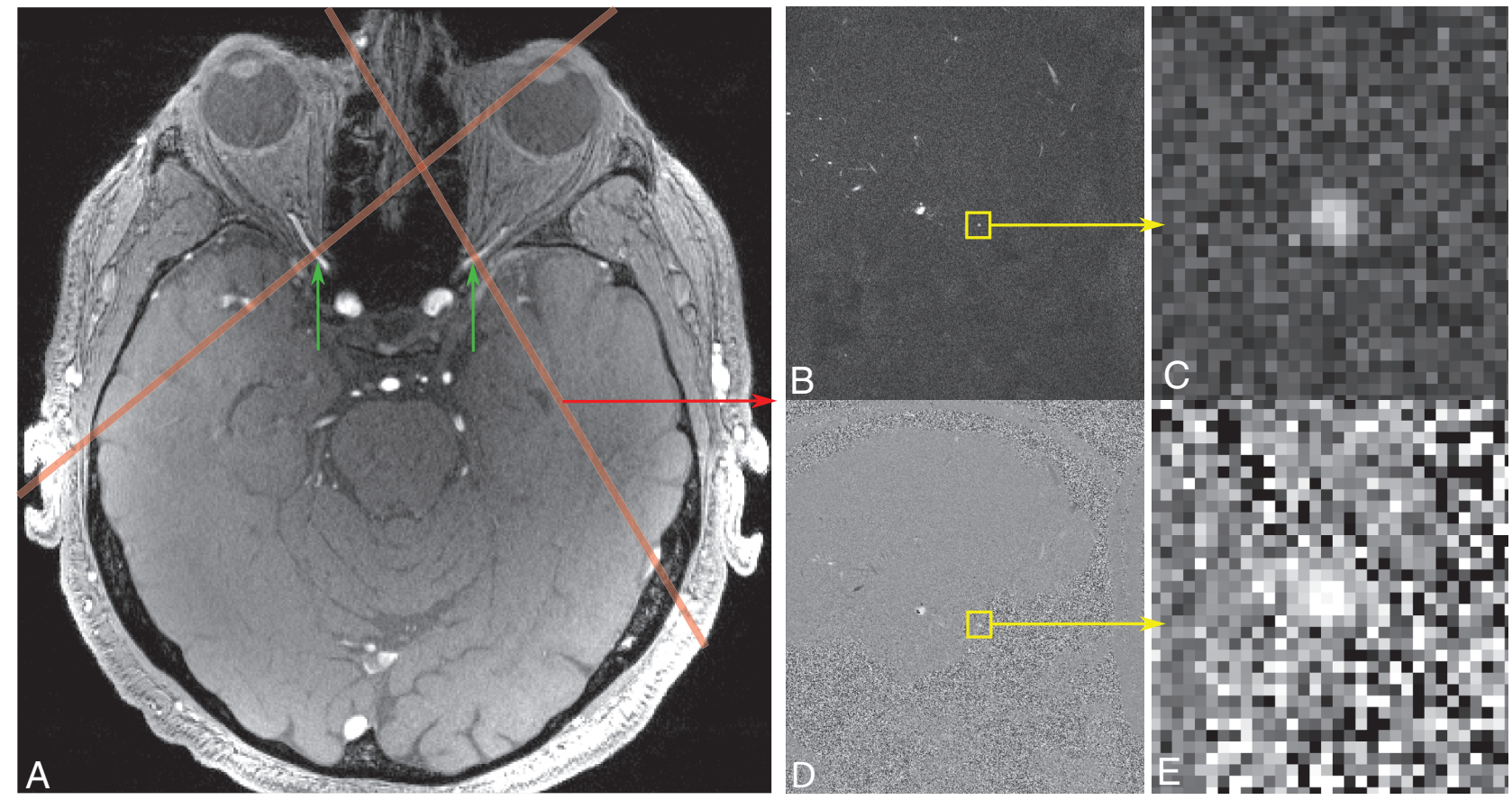

FIG 1. Positioning of PC-MRA slices using the 3D-TOF-MRA data as an anatomic guide. A, Red lines depict cross-sections of the triple-oblique positioning and location of PC-MRA slices centered on and perpendicular to each OA (green arrows), identified from the reference 3D-TOF image. The OA cross-section can be visualized in the center of the magnitude $(B$ and $C$ ) and phase $(D$ and $E)$ images from a PC-MRA scan (yellow box represents an enlarged region from $B$ and $D$ in $C$ and $E$, respectively).
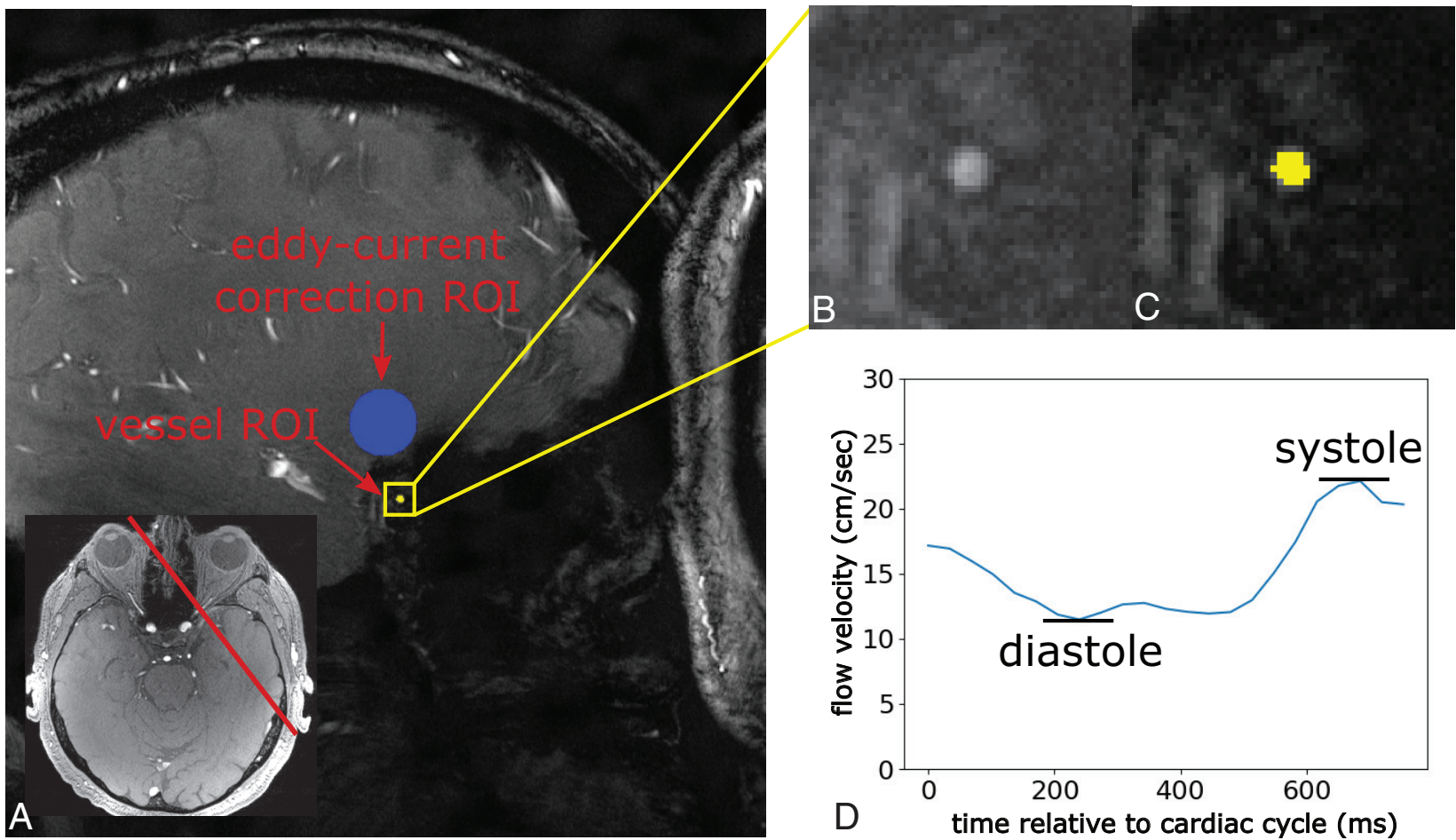

FIG 2. Analysis procedure for correcting $\mathrm{EC}$ bias and defining the vessel ROI. A, A PC-MRA magnitude image is used to place a 12 -mm-diameter $\mathrm{ROI}$ for EC correction in vessel-free tissue adjacent to the vessel ROI (blue). The red line in the inset TOF image indicates the PC-MRA slice position. The vessel ROI (yellow) is shown within the yellow box, which depicts the region enlarged in $B$ and $C$. Enlarged PC-MRA magnitude images show the vessel cross-section alone $(B)$ and with the vessel ROI superimposed $(C)$. $D$, Time-series of EC-corrected flow data in units of centimeters/second from 1 example PC-MRA acquisition with a single VENC value in 1 subject. 
Summary of vessel diameter and blood flow measures stratified by disease stage ${ }^{\text {a }}$

\begin{tabular}{|c|c|c|c|c|c|}
\hline & $\begin{array}{c}\text { Control (Mean) } \\
(\mathrm{SD})(n=20 \text { OA, } \\
24 \text { ICA) }\end{array}$ & $\begin{array}{c}\text { Early AMD (Mean) } \\
\text { (SD) }(n=9 \text { OA, } \\
15 \text { ICA) }\end{array}$ & $\begin{array}{c}\text { Interm. AMD } \\
\text { (Mean) (SD) } \\
\text { (n=7 OA, } 8 \text { ICA) }\end{array}$ & $\begin{array}{c}\text { Late AMD (Mean) } \\
\begin{array}{c}\text { (SD) }(n=12 \text { OA, } \\
17 \text { ICA) }\end{array}\end{array}$ & $\begin{array}{c}\text { All AMD (Mean) } \\
\text { (SD) }(n=28 \text { OA, } \\
40 \text { ICA })\end{array}$ \\
\hline OA diameter (mm) & $1.1(0.19)$ & $\begin{array}{r}0.9(0.25) \\
P=.11\end{array}$ & $\begin{array}{r}1.0(0.26) \\
P=.42\end{array}$ & $\begin{array}{r}0.8(0.25) \\
P<.01^{b}\end{array}$ & $\begin{array}{r}0.9(0.26) \\
P<.01^{b}\end{array}$ \\
\hline OA volume flow (mL/min) & $7.0(3.0)$ & $\begin{array}{c}6.5(4.2) \\
P=.74\end{array}$ & $\begin{array}{c}5.6(3.1) \\
P=.37\end{array}$ & $\begin{array}{c}3.8(1.8) \\
P<.01^{b}\end{array}$ & $\begin{array}{c}5.1(3.2) \\
P=.07\end{array}$ \\
\hline OA flow velocity $(\mathrm{cm} / \mathrm{s})^{c}$ & $13.1(4.0)$ & $\begin{array}{c}16.0(4.5) \\
P<.01^{b}\end{array}$ & $\begin{array}{c}11.9(4.3) \\
P=.91\end{array}$ & $\begin{array}{c}15.0(5.7) \\
P=.03^{\mathrm{b}}\end{array}$ & $\begin{array}{c}14.6(5.1) \\
P=.02^{\mathrm{b}}\end{array}$ \\
\hline OA RI & $0.70(0.10)$ & $\begin{array}{r}0.82(0.10) \\
P<.01^{\mathrm{b}}\end{array}$ & $\begin{array}{r}0.81(0.06) \\
P<.01^{\mathrm{b}}\end{array}$ & $\begin{array}{r}0.80(0.07) \\
P<.01^{\mathrm{b}}\end{array}$ & $\begin{array}{r}0.81(0.08) \\
P<.01^{\mathrm{b}}\end{array}$ \\
\hline ICA diameter (mm) & $4.3(0.51)$ & $\begin{array}{r}4.2(0.52) \\
P=.38\end{array}$ & $\begin{array}{r}4.6(0.63) \\
P=.47\end{array}$ & $\begin{array}{r}4.2(0.44) \\
P=.55\end{array}$ & $\begin{array}{r}4.3(0.52) \\
P=.67\end{array}$ \\
\hline ICA volume flow $(\mathrm{mL} / \mathrm{min})^{c}$ & $163.1(44.8)$ & $\begin{array}{c}154.3(44.2) \\
P=.98\end{array}$ & $\begin{array}{c}167.0(23.4) \\
P=.49\end{array}$ & $\begin{array}{c}166.6(27.7) \\
P=.19\end{array}$ & $\begin{array}{c}162.2(33.8) \\
P=.46\end{array}$ \\
\hline ICA flow velocity $(\mathrm{cm} / \mathrm{s})^{c}$ & $18.3(3.5)$ & $\begin{array}{r}18.7(3.7) \\
P=.07\end{array}$ & $\begin{array}{r}17.8(4.5) \\
P=.58\end{array}$ & $\begin{array}{r}20.6(5.2) \\
P<.01^{\mathrm{b}}\end{array}$ & $\begin{array}{c}19.4(4.6) \\
P<.01^{b}\end{array}$ \\
\hline ICA RI & $0.61(0.09)$ & $\begin{array}{c}0.66(0.09) \\
P=.18 \\
\end{array}$ & $\begin{array}{r}0.70(0.04) \\
P<.01^{b} \\
\end{array}$ & $\begin{array}{r}0.65(0.11) \\
P=.29 \\
\end{array}$ & $\begin{array}{c}0.67(0.09) \\
P=.07 \\
\end{array}$ \\
\hline
\end{tabular}

Note:-RI indicates resistance index; Interm., intermediate.

${ }^{a}$ Values represent group mean and group SD, and reported $P$ values indicate significance relative to controls based on a GEE analysis followed by post hoc least significant difference comparisons between disease groups and controls. The sample size for ophthalmic artery measurements was 48 hemisphere measurements from 30 subjects. The sample size for the ICA measurements was 64 hemispheres of 33 subjects.

${ }^{\mathrm{b}}$ Tests that were significant at the $P<.05$ level (uncorrected).

${ }^{c}$ Adjusted for age, which was statistically significant $(P<.05)$ in the GEE model.

estimated and corrected on a per-scan basis by placing a $12-\mathrm{mm}$ diameter circular ROI in tissue devoid of visible vessels adjacent to each vessel ROI (Fig 2), similar to a previously described correction method. ${ }^{14-16}$ The average phase within this ROI was subtracted from the phase image before further analysis. The average phase offset in the adjacent tissue ROIs corresponding to the OA was -0.01 radians for patients with AMD and -0.04 radians for controls $(P>.05)$, and in the ROIs corresponding to the ICA, it was 0.25 radians for patients with AMD and 0.21 radians for controls $(P>.05)$.

\section{Flow Phantom Measurements}

A custom-built flow phantom was used to validate PC-MRA measurements and the data-analysis procedure. Briefly, the tubing of the flow phantom was wrapped twice around a spherical agar gel phantom and placed in the receive coil. The flow phantom consisted of a continuous circuit of water driven by a constant-flow-rate pump with a controllable flow rate attached to a flowmeter rated at $\pm 4 \%$ accuracy (McMaster-Carr part number $5079 \mathrm{k} 54)$. The MR imaging acquisition procedure described above was followed. Phantom data were analyzed with and without correction of EC biases. Flow phantom measurements with the phantom set to a flow rate of $20 \mathrm{~cm} / \mathrm{s}$, similar to the mean flow rate through the ICAs, indicated that the quantitative accuracy of the flow measurement was improved with the EC correction and was, on average, better than $0.8 \mathrm{~cm} / \mathrm{s}$ after correction (Online Supplemental Data).

\section{MR Imaging Data Analysis: Morphometry and Flow Quantification}

PC-MRA data were analyzed using the data from the lowest VENC available, which either did not result in phase aliasing or were able to be unaliased. Vessel ROIs were defined manually in a 2-step process using both magnitude-valued intensities and velocity-weighted phase-valued data from each VENC. First, candidate voxels were identified from the magnitude-valued image; then, this grouping of voxels was refined using the dynamic velocity-weighted data to select those candidate voxels exhibiting a clear change in velocity between the systolic and diastolic phases (Fig 2). Portions of voxel-specific time-series with aliased phase data were identified by comparing the sign of the phase value with that in the time-series of a larger VENC, which was manually confirmed to not contain aliased phase data. These aliased portions of phase data were then unwrapped before further analyses.

The area of all voxels in the vessel ROI was calculated and used to convert between linear flow velocity $(U)$ and volumetric flow rate $(Q)$ according to the formula: $Q=U \times$ Vessel Area. Flow measurements from all voxels within the vessel ROI at each time point were averaged to obtain a mean vessel flow timeseries, which was then temporally filtered using a 3-point mean kernel, yielding an effective temporal resolution of approximately $100 \mathrm{~ms}$. These filtered data were used in all further analyses.

$\mathrm{OA}$ and ICA flow properties were quantified using four parameters:

1) Mean flow rates were calculated across the filtered timeseries covering a complete cardiac cycle.

2) The maximum systolic velocity $\left(U_{\text {syst }}\right)$ and volumetric flow $\left(Q_{\text {syst }}\right)$ and minimum diastolic velocity $\left(U_{\text {dias }}\right)$ and volumetric flow $\left(Q_{\text {dias }}\right)$ were obtained from the filtered time-series. The resistance index (RI) was calculated as the ratio of the difference between $U_{\text {syst }}$ and $U_{\text {dias }}$ to $U_{\text {syst: }}$ :

$$
\mathrm{RI}=\frac{U_{\text {syst }}-U_{\text {dias }}}{U_{\text {syst }}}
$$

3) OA flow rates were normalized to ipsilateral ICA flow rates. 

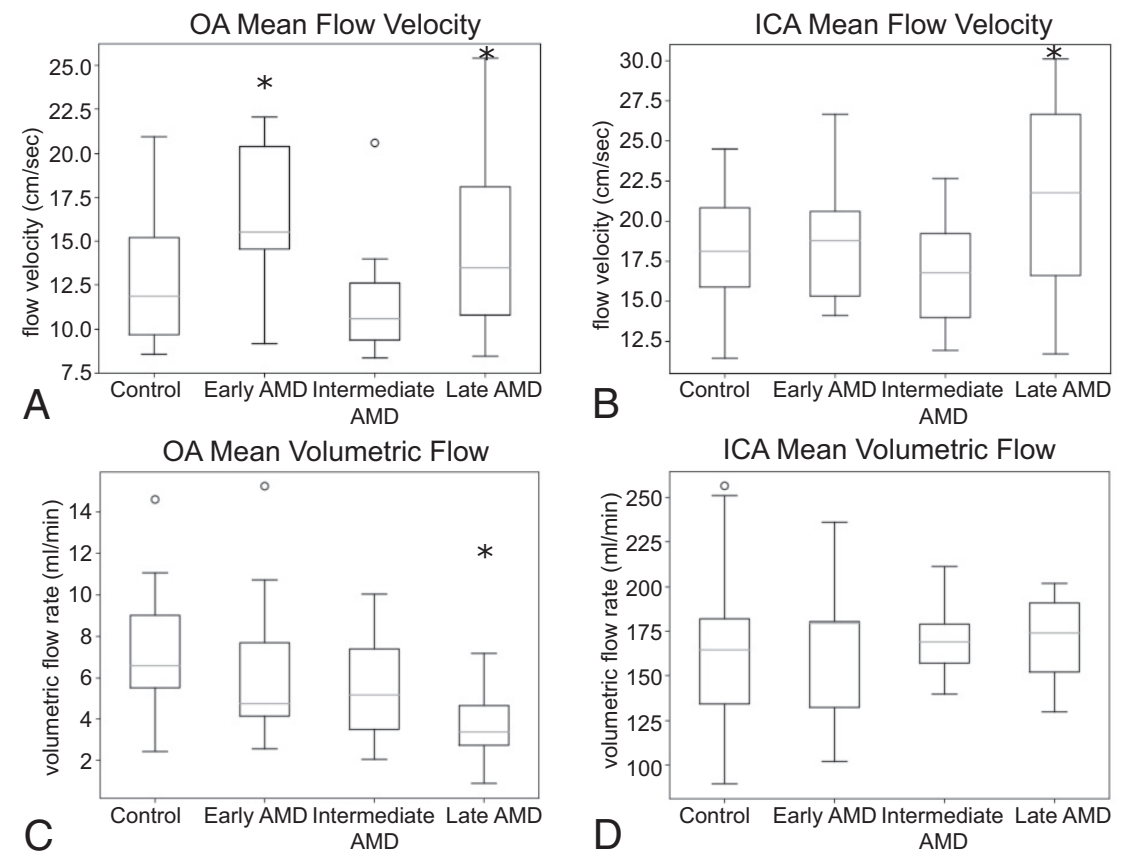

FIG 3. Boxplots of unnormalized flow velocities $(A$ and $B)$ and volumetric flow rates $(C$ and $D)$ in the OAs ( $A$ and $C$ ) and ICAs ( $B$ and $D)$. Vessels were divided into 4 groups: healthy controls, early AMD, intermediate AMD, and late AMD. The asterisk signifies $P<.05$ compared with controls. measurements, respectively, and fitting this code in the GEE model as a covariate. Age was included in the GEE model as well as calculation of post hoc comparisons if statistically significant. The GEE approach also has the advantage of leaving the estimates of the group means unchanged for those variables for which age did not significantly influence the measurements, simplifying interpretation. A $P$ value $<.05$ was considered statistically significant, and $P$ values were not adjusted for multiple comparisons. Reported values are expressed as mean (SD).

\section{RESULTS}

Thirty-seven subjects (24 patients with AMD; 14 women and 10 men; mean age, 77.8 [SD, 8.2] years; and 13 controls; 8 women and 5 men; mean age, $69.3[\mathrm{SD}, 7.4]$ years) were enrolled in the study. Data from 16 patient OAs, 6 patient ICAs, 6 control OAs, and 2 control ICAs were discarded before quanti-

\section{OA Mean Volumetric Flow Trends}

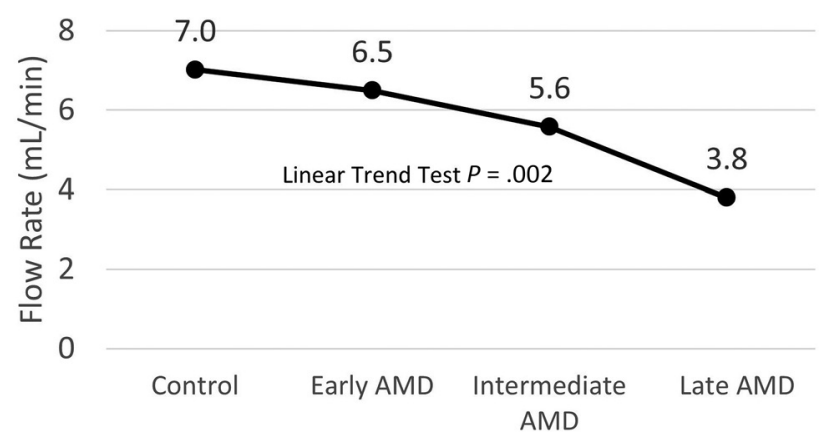

FIG 4. Linear trend test plot, which demonstrates that the rate of decline in OA volumetric flow with disease progression is statistically significant.

4) OA and ICA inner diameters were estimated by assuming that the vessel cross-section is a circular disc, calculating the cross-sectional area of the vessel ROI, and then estimating the diameter of the circle on the basis of this area.

\section{Statistical Analysis}

Because measurements from both hemispheres of some patients and controls were included in the statistical analysis, we used the generalized estimating equation (GEE) method with a linear link function to account for correlated data. ${ }^{17}$ Patient group means were compared with those of controls with post hoc least significant difference tests. Trends in group means were assessed by assigning an ordinal code from 0 through 3 to the healthy controls, early AMD, intermediate AMD, and late AMD fying flow rates due to subject motion. In addition, data from 1 patient with atrial fibrillation were not usable, and 2 patients had AMD diagnosed in only 1 eye, and for these, only the OA corresponding to the eye with AMD was included in our analyses. In total, data from 28 OAs and 40 ICAs from 21 patients with AMD and 20 OAs and 24 ICAs from 12 healthy similarly-aged controls were included in the analyses. In analyses of OA flow rates normalized to upstream ICA flow rates, only OA-ICA pairs in which data from both vessels were usable were included, resulting in 28 pairs from patients with AMD (9 early AMD, 7 intermediate $\mathrm{AMD}$, and 12 late $\mathrm{AMD}$ ) and 20 pairs from healthy similarlyaged controls. The late AMD group included both late dry AMD and wet AMD.

Mean flow velocities differed between controls and patients in both the OA and the ICA. Mean volumetric flow rates through the $\mathrm{OA}$ were only significantly lower in patients with late $\mathrm{AMD}$ relative to controls, while volumetric flow rates through the ICA were similar between groups (Table and Fig 3). Furthermore, there was a statistically significant trend of decreasing volumetric flow rates through the $\mathrm{OA}$ as disease severity increased from the early to late stage ( $P=.002$, GEE test of trend in means, Fig 4). In 3 subjects with intermediate stage dry AMD in one eye and wet AMD in the other eye, this pattern was maintained; the OA on the side with wet AMD had a volumetric flow rate at least $1.6 \mathrm{~mL} / \mathrm{min}$ lower than that on the side with intermediate dry AMD. This difference in volumetric flow rates was mainly driven by reduction in the OA diameter in patients with $\mathrm{AMD}(P<.05$, Table). The right $\mathrm{OA}$ was scanned twice in 1 subject using identical acquisition parameters and analyzed independently; the mean volumetric flow rates agreed within $10 \%$, while the vessel ROI (and hence the diameter) was identical across the two scans. This low discrepancy of $10 \%$ 
between scans (albeit based on 1 repeat scan in 1 subject) was substantially less than the difference observed between the controls and the patients with AMD overall (27\%) and the
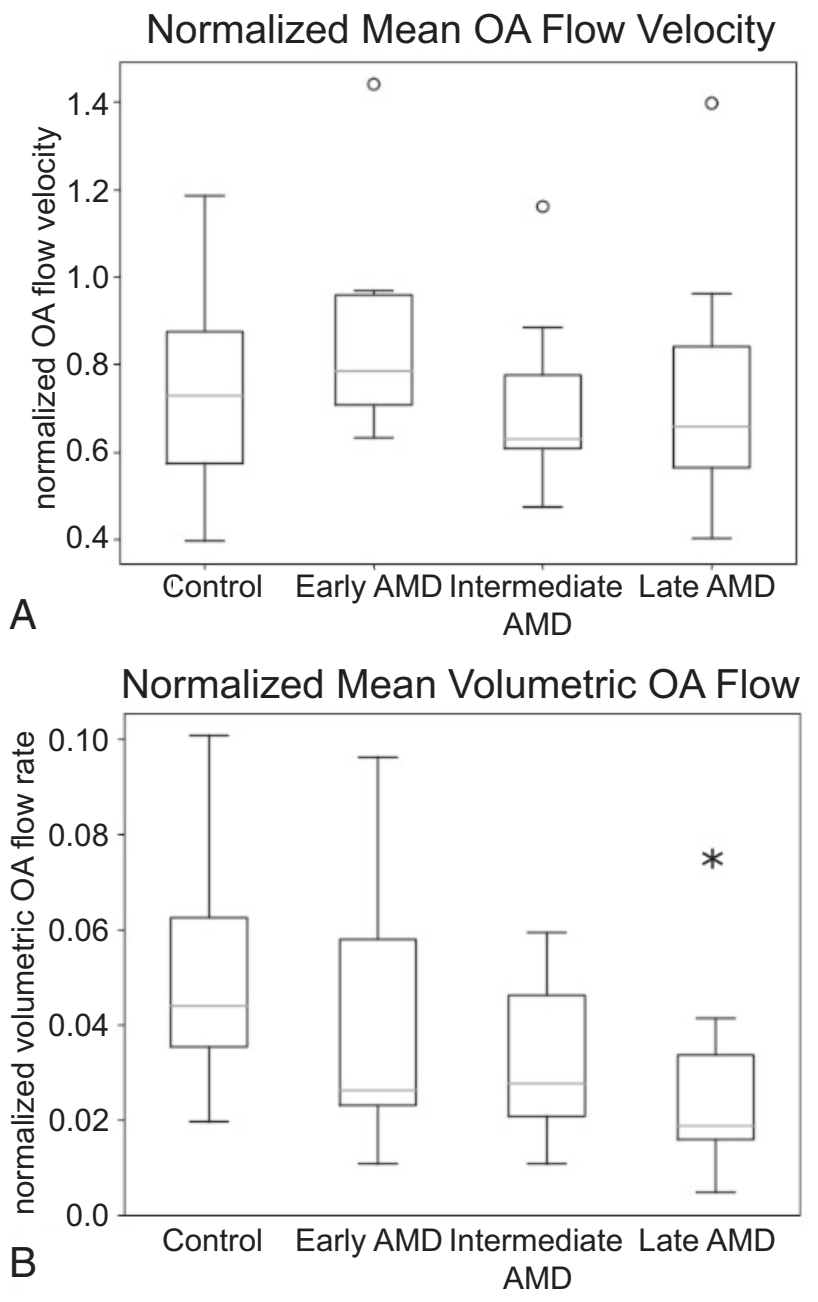

FIG 5. Boxplots of flow velocity $(A)$ and volumetric flow $(B)$ in the OA normalized to upstream ICA flow rates. Normalized flow values represent the OA flow as a ratio of ICA flow. Vessels are divided into 4 groups: healthy controls, early AMD, intermediate AMD, and late AMD. The asterisk signifies $P<.05$ compared with controls. late AMD group (46\%), suggesting that our measurement precision is sufficient to detect these group-level differences.

The difference in flow rates is further elucidated by normalizing the OA flow to the upstream ICA flow on a per-vessel basis; normalized volumetric OA flow in controls is nearly double that of patients on average $(P<.01$, GEE test of trend in means, Fig $5 A$ ). The resistance index was calculated to capture blood flow dynamics over the cardiac cycle and was significantly higher compared with controls in the OA in the overall group of patients with AMD, but only in the patient group with intermediate stage AMD in the ICA $(P<.05$, Table and Fig 6$)$.

\section{DISCUSSION}

While previous research has found that flow velocities in the choroid $^{5}$ and retina ${ }^{18}$ do not differ between controls and patients but volumetric flow rates do, our study found both significantly higher flow velocities in the OA and ICA in patients compared with controls, as well as significantly lower volumetric flow in the $\mathrm{OA}$ in patients compared with controls. These results suggest that vascular changes may potentially begin upstream of the choroid. Our data report only an association and cannot establish causality. It is possible that the reduced upstream blood flow may be caused instead by reduced metabolic demand from retinal degradation. Future studies may seek to clarify the causal relation between reduced upstream blood flow and AMD. Our results do, however, suggest a mechanism for the reduced choroidal perfusion, which is thought to be a contributing factor to the development of late AMD. ${ }^{6}$

To our knowledge, the finding that OA diameters are decreased in patients with AMD has not previously been reported. The underlying cause of this decrease bears further investigation. Our use of PC-MRA images to measure the inner vessel diameters could introduce error because the diameter measurement could be impacted by flow velocities. If flow velocities were lower, the diameter measurement could be underestimated. However, we found a statistically significant trend in which flow velocities increase slightly as vessel diameter decreases, suggesting that our estimates of smaller vessel diameters are not due to this potential bias. In addition, the true spatial resolution of the PC scan could affect the accuracy of our vessel diameter measurements. However, we found that the true spatial resolution is negligibly different from the voxel size; the expected blurring due to $\mathrm{T} 2{ }^{*}$ decay during each gradient-echo line readout of duration, $5 \mathrm{~ms}$, was $<4 \%$.

For our flow measurements, we used a single velocity encoding direction, and care was taken to angle the PC-MRA slice perpendicular to the OA. If, however, a slice were imperfectly positioned, this would result in a biased blood velocity estimate that was lower than the true velocity. For a large obliquity of $25^{\circ}$, this would result in an underestimate of about $10 \%$ in absolute velocity. ${ }^{16}$ Perhaps if the $\mathrm{OA}$ were more curved in patients with AMD, there might be more obliquity leading to an underestimation of
FIG 6. Resistance indices for the OA $(A)$ and ICA $(B)$. Vessels are divided into 4 groups: healthy controls, early $A M D$, intermediate AMD, and late AMD. The asterisk signifies $P<.05$ compared with controls.

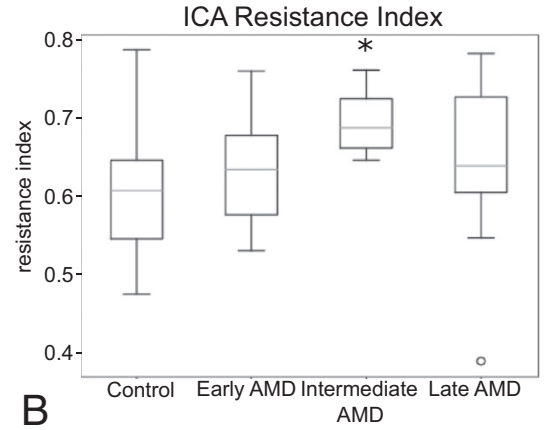

1658

Hibert Sep $2021 \quad$ www.ajnr.org 
flow velocity in these patients, but we found the opposite: Flow velocity was found to be higher in patients with AMD. In addition, any systematic obliquity in patients with AMD would have led to an increase in the OA diameter in these patients, but we found decreased OA diameters. Nevertheless, it seems most likely that any obliquity, if it is present, is small and is unlikely to be systematically different between groups. Therefore, this effect likely contributes to the variance across subjects but not to bias across groups.

Further limitations of this study include the overall small sample size and the younger average age of the control population. AMD is a complex genetic disease associated with a wide range of systemic comorbidities such as cardiovascular disease, hypertension, obesity, and nutritional deficiencies. While larger future studies are needed to investigate the effects of these comorbidities on OA and ICA blood flow, this limitation does not diminish the importance of our imaging strategy and findings of abnormalities in blood flow. Additionally, our data are in close agreement with flow measures through the OA reported recently in healthy elderly subjects ( $n=84$ eyes) based on lower-resolution data at $3 \mathrm{~T}^{15}$ this previous study reported similar flow velocities and volumetric flow rates and a resistance index of 0.68 (SD, 0.08) compared with our value of 0.70 (SD, 0.10) in similarly-aged control subjects.

While quantifying flow through the OA was the primary goal of this study, flow through the ICA was also investigated to differentiate $\mathrm{OA}$ flow changes from any systemic flow alterations. The increase in the resistance index in both the ICA and OA combined with the decreased OA volumetric flow as a percentage of ICA volumetric flow suggests that there may be both systemic and local vascular changes associated with AMD. These metrics could not be evaluated using only a vessel-diameter measurement; dynamic flow measurements were necessary to detect these differences between patients with AMD and controls.

\section{CONCLUSIONS}

Our findings of structural and flow differences in the vasculature of patients with AMD compared with healthy controls indicate that changes in vasculature upstream of the eye are associated with AMD. If, motivated by our finding, future studies can establish a causal link between these upstream changes and AMD, it may raise the possibility of treatment strategies targeting the $\mathrm{OA}$ to restore blood flow to the retina in patients with AMD.

\section{ACKNOWLEDGMENTS}

The authors would like to thank Dr Bastien Guérin for providing the flow phantom used to validate the PC-MRA measurements, Siemens for providing WIP 452B, and Dr Himanshu Bhat for technical assistance with the quantitative PC-MRA pulse sequence.

Disclosures: Matthew L. Hibert—RELATED: Grant: OcuDyne Inc.* Yinching Iris ChenRELATED: Grant: OcuDyne, Comments: study sponsor.* Nadia K. Waheed-RELATED: Grant: OcuDyne, Comments: My institution was a clinical trial site for the study and, as such, received reimbursement for services provided to subjects in the process of screening for eligibility*; UNRELATED: Employment: Gyroscope Therapeutics,
Comments: employee at Gyroscope, a clinical stage gene therapy company; Stock/ Stock Options: OcuDyne. Jeffrey S. Heier-UNRELATED: Board Membership: Ocular Therapeutix, Comments: member of the Board of Directors; Consultancy: 4D Molecular Therapeutics, Adverum Biotechnologies, Aerie, Aerpio Pharmaceuticals, Aldeyra Therapeutics, Allegro, Alzheon, Annexon Biosciences, Apellis Biosciences, Aprea, AsclepiX Therapeutics, Aviceda Therapeutics, Bionic Vision Technologies, Dark Horse, DTx Pharma, Eloxx Pharmaceuticals, Galimedix Therapeutics, Genentech, Graybug Vision, Gyroscope, Iveric Bio, jCyte, Chengdu Kanghong Pharmaceutical Group, LensGen, NM Novartis, Ocular Therapeutix, OcuTerra, Oxurion, Palatinechnologies, Regeneron Pharmaceuticals, Regenxbio, Stealth, Thea, Verseon, Vinci, Voyant, Comments: I serve as scientific consultant for these companies; Grants/Grants Pending: Apellis Pharmaceuticals, Asclepix, Bayer, Genentech, Gyroscope, Hemera, Iveric, Chengdu Kanghong Pharmaceutical Group, Kodiak Sciences, Notal Vision, Novartis, Regeneron, Regenxbio, Stealth, Comments: I serve as Principal Investigator for clinical trials with these sponsors*; Stock/Stock Options: Adverum, Aldeyra, Allegro, Aviceda, Digital Surgery Solution, DTx Pharma, jCyte, Ocular Therapeutix, Vinci, Comments: stock or stock options for scientific consulting services. Michael W. Calhoun—UNRELATED: Employment: OcuDyne Inc, Comments: I currently serve as Vice President of Research for OcuDyne Inc. Philip R. RosenfeldUNRELATED: Consultancy: OcuDyne, Comments: consulting relationship with OcuDyne; Stock/Stock Options: OcuDyne, Comments: stock options that have not been exercised. Jonathan R. Polimeni-RELATED: Grant: OcuDyne, Comments: grant contract managed by Massachusetts General Hospital (as indicated in the article)*; Payment for Writing or Reviewing the Manuscript: OcuDyne, Comments: payment for finalizing manuscript after the end of the grant period. William J. FeuerRELATED: Personal Fees: OcuDyne, Comments: fee for statistical consulting/analysis. *Money paid to the institution.

\section{REFERENCES}

1. Ling Wong W, Su X, Li X, et al. Global prevalence of age-related macular degeneration and disease burden projection for 2020 and 2040: a systematic review and meta-analysis. Lancet Glob Heal 2014;2:e106-16 CrossRef Medline

2. Ratnapriya R, Chew EY. Age-related macular degeneration-clinical review and genetics update. Clin Genet 2013;84:160-16 CrossRef Medline

3. Ferris FL, Wilkinson CP, Bird A, et al. Clinical classification of agerelated macular degeneration. Ophthalmology 2013;120:844-51 CrossRef Medline

4. Dry vs Wet Age-Related Macular Degeneration. AMDF. https:// www.macular.org/dry-vs-wet-macular-degeneration. Accessed November 2, 2019

5. Boltz A, Luksch A, Wimpissinger B, et al. Choroidal blood flow and progression of age-related macular degeneration in the fellow eye in patients with unilateral choroidal neovascularization. Invest Ophthalmol Vis Sci 2010;51:4220-25 CrossRef Medline

6. Metelitsina TI, Grunwald JE, DuPont JC, et al. Foveolar choroidal circulation and choroidal neovascularization in age-related macular degeneration. Invest Ophthalmol Vis Sci 2008;49:358-63 CrossRef Medline

7. Dai W, O'Loughlin L, Yu G, et al. Arterial spin labeling measured choroidal blood flow is reduced in age-related macular degeneration and correlates with severity level. Proc Intl Soc Mag Reson Med 2016;24:1416

8. Grunwald JE, Metelitsina TI, DuPont JC, et al. Reduced foveolar choroidal blood flow in eyes with increasing AMD severity. Invest Ophthalmol Vis Sci 2005;46:1033-38 CrossRef Medline

9. Chaine G, Hullo A, Sahel J, et al. Case-control study of the risk factors for age related macular degeneration. $\mathrm{Br} J$ Ophthalmol 1998;82:996-1002 CrossRef Medline

10. Stamm AC, Wright CL, Knopp M, V, et al. Phase contrast and timeof-flight magnetic resonance angiography of the intracerebral arteries at 1.5, 3 and 7 T. Magn Reson Imaging 2013;31:545-49 CrossRef Medline

11. Kang CK, Park CA, Lee DS, et al. Velocity measurement of microvessels using phase-contrast magnetic resonance angiography at 7 Tesla MRI. Magn Reson Med 2016;75:1640-46 CrossRef Medline

12. Keil B, Triantafyllou C, Hamm M, et al. Design optimization of a 32channel head coil at 7T. In: Proceedings of the International Society of Magnetic Resonance in Medicine, Stockholm, Sweden. May 1-7, 2010 
13. Hayreh SS, Dass R. The ophthalmic artery, I: origin and intra-cranial and intra-canalicular course. Br J Ophthalmol 1962;46:65-98 CrossRef Medline

14. Pelc NJ, Sommer FG, Li KC, et al. Quantitative magnetic resonance flow imaging. Magn Reson Q 1994;10:125-17 Medline

15. Ambarki K, Hallberg P, Jóhannesson G, et al. Blood flow of ophthalmic artery in healthy individuals determined by phase-contrast magnetic resonance imaging. Invest Ophthalmol Vis Sci 2013;54:2738-45 CrossRef Medline
16. Bernstein MA, King KF, Zhou XJ. Handbook of MRI Pulse Sequences. Academic Press; 2004

17. Ying G-S, Maguire MG, Glynn R, et al. Tutorial on biostatistics: linear regression analysis of continuous correlated eye data. Ophthalmic Epidemiol 2017;24:130-40 CrossRef Medline

18. Ciulla TA, Harris A, Chung HS, et al. Color Doppler imaging discloses reduced ocular blood flow velocities in nonexudative agerelated macular degeneration. Am J Ophthalmol 1999;128:75-80 CrossRef Medline 